\title{
REVISIÓN DE TEMA
}

\section{Triatoma dimidiata en Colombia; distribución, ecología e importancia epidemiológica}

\author{
Óscar Quirós-Gómez ${ }^{1,2}$, Nicolás Jaramillo³, Víctor M. Angulo,4, Gabriel Parra-Henao',5 \\ 1 Red Chagas, Bogotá, D.C., Colombia \\ Grupo de Epidemiología y Bioestadística, Universidad CES, Medellín, Colombia \\ 3 Instituto de Biología, Universidad de Antioquia, Medellín, Colombia \\ 4 Centro de Investigación en Enfermedades Tropicales, CINTROP, Universidad Industrial de Santander, \\ Bucaramanga, Colombia \\ 5 Centro de Investigación en Salud para el Trópico, Universidad Cooperativa de Colombia, Santa Marta, Colombia \\ Triatoma dimidiata es un importante vector de la enfermedad de Chagas en Centroamérica y en los \\ países del norte de Suramérica. En Colombia, tiene una amplia distribución y su presencia se ha \\ reportado en 14 departamentos de las regiones Andina, Caribe, de los Llanos Orientales y del Alto \\ Magdalena, en las cuales ocupa diferentes ecótopos naturales y artificiales. \\ La especie está clasificada como secundaria para la transmisión de Trypanosoma cruzi; sin embargo, \\ su presencia en el ambiente silvestre, peridoméstico y doméstico en la región Andina, y su capacidad \\ de movilizarse entre ellos, le han permitido escapar al control basado en la aspersión con piretroides \\ y ello resalta su importancia en el mantenimiento de la transmisión del parásito debido a la potencial \\ reinfestación de las viviendas. \\ La comprensión de las relaciones de $T$. dimidiata con su hábitat, así como el empoderamiento de la \\ comunidad, contribuyen al desarrollo de sistemas de control efectivos y perdurables. \\ El propósito de esta revisión fue describir la distribución, los factores de riesgo, la ecología, las \\ características entomológicas y los hábitats de las poblaciones de T. dimidiata en Colombia, y proponer \\ alternativas de intervención acordes con las características particulares de esta especie.
}

Palabras clave: Triatoma; Trypanosoma cruzi; enfermedad de Chagas; epidemiología; ecología. doi: http://dx.doi.org/10.7705/biomedica.v37i2.2893

\section{Triatoma dimidiata in Colombia: Distribution, ecology and epidemiological importance}

Triatoma dimidiata is an important vector of Chagas disease in Central America and countries of northern South America. In Colombia, it has a wide geographical distribution with reported presence in 14 departments in the Andean, Caribbean, Eastern plains and Upper Magdalena regions, where it occupies different natural and artificial ecotopes.

The species is considered a secondary vector in the transmission of Trypanosoma cruzi. Its presence in wild, peridomestic and intradomiciliary habitats in the Andean region, coupled with its ability to move between them, has allowed it to escape the control based on pyrethroids spraying, highlighting its importance in maintaining transmission of the parasite through the potential reinfestation of homes. Understanding the relation of $T$. dimidiata and its habitats, as well as the empowerment of communities, will contribute to the development of effective and lasting control systems.

The purpose of this review was to describe the distribution, risk factors, ecology, entomological features and habitats of $T$. dimidiata populations in Colombia, and to propose alternative interventions in agreement with the specific characteristics of the species.

Key words: Triatoma; Trypanosoma cruzi; Chagas disease; epidemiology; ecology. doi: http://dx.doi.org/10.7705/biomedica.v37i2.2893

En esta revisión se resume la información conocida hasta la fecha sobre la distribución, ecología e importancia epidemiológica deTriatoma dimidiata, vector de la enfermedad de Chagas en Colombia.

La enfermedad de Chagas es la tercera enfermedad parasitaria más común a nivel global después de la malaria y la esquistosomiasis, y se considera un importante problema de salud pública en

\footnotetext{
Contribución de los autores:

Óscar Quirós-Gómez: concepción y diseño del estudio, recolección y resumen de información bibliográfica y escritura del manuscrito Nicolás Jaramillo: recolección de información bibliográfica y escritura del manuscrito

Víctor M. Angulo: análisis e interpretación de datos

Gabriel Parra-Henao: concepción y diseño del estudio, análisis e interpretación de datos y escritura del manuscrito

Todos los autores participaron en la revisión del manuscrito.
} 
Latinoamérica $(1,2)$. A pesar de ser catalogada por la Organización Mundial de la Salud (OMS) como una de las 17 enfermedades tropicales desatendidas, principalmente por su asociación con la pobreza, no se la considera una prioridad en los programas de salud pública (3). Dicha desatención puede verse exacerbada por el impacto de enfermedades emergentes como la infección por el virus del zika, la cual recientemente ha llegado a las grandes concentraciones urbanas y, además de sus síntomas en la fase aguda, se ha asociado con el síndrome de Guillain-Barré $(4,5)$ y con microcefalia en recién nacidos (6). Tales efectos la han situado bajo el foco de máxima atención de la población y de los medios de comunicación, lo cual podría ir en detrimento de los programas de vigilancia y control de la enfermedad de Chagas, a pesar del profundo efecto que esta tiene en las comunidades rurales más desfavorecidas del país.

La más reciente estimación de la magnitud de la enfermedad se resume en los indicadores de la Organización Panamericana de la Salud (OPS) para el año 2010: el número de infectados en el país para la fecha era de 437.960 , la tasa de prevalencia era de 0,96\%, con cerca de 4'813.543 personas en riesgo de adquirir la infección (3). Las vías de transmisión son diversas: la congénita, la oral, la transfusión de hemocomponentes contaminados con el parásito $\mathrm{y}$, menos frecuente, el accidente laboral. Sin embargo, la transmisión vectorial es la de mayor magnitud y más difícil control, con una incidencia anual de 5.274 casos (3).

Colombia tiene una rica fauna de triatominos, con presencia de 26 de las 144 especies conocidas, de las cuales 15 se han hallado con infección natural por Trypanosoma cruzi. De Rhodnius prolixus, la principal especie vectora debido a su eficiencia en la transmisión del parásito, se han detectado poblaciones introducidas en la región Andina, y autóctonas en la región de la Orinoquia (7). Triatoma dimidiata Latreille, 1811 (Hemiptera: Reduviidae: Triatominae) es considerada la segunda especie vectora dada su frecuencia en las viviendas humanas, la tasa de infección natural con $T$. cruzi y su amplia dispersión en la geografía colombiana. T. dimidiata se halla en gran diversidad de ecótopos, con reporte de diferencias intraespecíficas que sugieren la

\footnotetext{
Correspondencia:

Gabriel Parra-Henao, Carretera Troncal del Caribe, Km 3 Sector Mamatoco, Santa Marta, Colombia

Teléfono: (575) 420 9604, extensión 5538

gparrahenao@gmail.com
}

Recibido: 03/07/16; aceptado: 19/07/16 presencia de varias poblaciones y acentuados contrastes en su relación con los hábitats humanos, por lo cual su perfil epidemiológico tiene alcance diferencial $(8,9)$.

En algunos reportes sobre el riesgo epidemiológico en el país se ha recomendado ahondar en la comprensión del proceso de domiciliación de $T$. dimidiata, ya que tiene presencia en zonas selváticas y, además, invade las viviendas humanas. El estudio de su ecología y comportamiento en el ambiente natural permitiría el desarrollo de nuevas estrategias de vigilancia (10). Reyes, et al. (2007), Farfan, et al. (2011), y Parra-Henao, et al., han resaltado la importancia de realizar estudios más amplios de las características particulares de $T$. dimidiata, principalmente de la población presente en los departamentos de Boyacá y Santander, debido a que la fumigación con piretroides ha resultado poco efectiva por su movilidad entre el área silvestre, el peridomicilio y el domicilio, así como por su gran capacidad de invadir las viviendas y la baja sensibilidad demostrada en ensayos de laboratorio $(11,12)$.

\section{Distribución}

\section{América Latina}

Triatoma dimidiata se encuentra distribuida en una amplia región del continente americano que va desde México hasta el norte de Perú (13). En este amplio territorio se la puede encontrar en hábitats silvestres, en el peridomicilio y en el domicilio, y ha habido reportes de su presencia en el área urbana en diferentes países de América Central, así como en Colombia y en Ecuador (14-17). Con frecuencia se reportan poblaciones en los tres hábitats en una misma área geográfica, en tanto que en Belice, por ejemplo, solo se la ha encontrado en condición silvestre y, ocasionalmente, en el peridomicilio (18).

Las poblaciones domésticas raramente son numerosas; no obstante, la especie se considera el vector más importante de la enfermedad de Chagas en Costa Rica, Ecuador, Guatemala, El Salvador y Nicaragua, y un importante vector secundario en Colombia y Honduras (19). En Ecuador y en el norte de Perú, $T$. dimidiata probablemente fue introducida por vía marítima en la época precolombina y se encuentra exclusivamente en el domicilio y el peridomicilio (20).

La información genética y morfológica sugiere que $T$. dimidiata pudo originarse en la península de Yucatán y, desde allí, dispersarse hacia el sur y el norte del continente, diferenciándose en 
poblaciones relativamente discretas $(20,21)$. Se ha sugerido que en la base de la península de Yucatán se ubicó un centro de dispersión que dio origen a dos linajes evolutivos principales: uno restringido a la península, incluido Belice y el norte de Guatemala, en el cual se originó $T$. spp. affinis dimidiata, y el otro que se dispersó a través de Centroamérica hasta Colombia, y hacia el norte hasta el centro de México y la isla de Cozumel donde se halla la forma insular $T . d$. hegneri. Este linaje dio origen a las tres subespecies continentales del complejo: $T$. d. maculipennis en México, $T$. $d$. dimidiata desde Centroamérica hasta Costa Rica (y Ecuador, a donde llegó por dispersión pasiva) y $T$. d. capitata desde Panamá hasta Colombia (22). Asimismo, recientemente se ha conocido que en la península de Yucatán existe una amplia zona de hibridación natural de $T$. affin a dimidiata y $T$. d. maculipennis, lo cual confirma la estrecha relación filogenética entre estos dos taxones (23) (figura 1).

\section{Triatoma dimidiata en Colombia}

La especie fue reportada en el país por Usinger, et al., 1941 (24), Otálora, 1952 (25), Ucrós, et al., 1960 (26), Marinkelle, 1969 (27), D’Alessandro, et al.,1981 (28) , Zeledón, et al., 1981 (29), Corredor, et al, 1990 (30), Restrepo, et al., 2002 (31), Guhl, et al, 2007 (7), y Parra-Henao, et al., 2010 (32) y 2015 (33). Se distribuye desde La Guajira hasta Huila, cubriendo 14 departamentos, incluidas las islas de San Andrés y Providencia en el mar Caribe.

Triatoma dimidiata presenta un amplio rango altitudinal: en la región Caribe, en el Urabá antioqueño, en el departamento de Magdalena y en La Guajira, entre los 10 y los 40 metros sobre nivel del mar (msnm); en los municipios de las llanuras del departamento del Cesar, entre los 40 y los 175 msnm; en los municipios de los departamentos del Huila, Meta y Boyacá en las estribaciones de la Cordillera Oriental, y en los Llanos Orientales, en altitudes desde los 412 hasta los $488 \mathrm{msnm}$; en los valles interandinos de Boyacá y Santander alcanza los 620 a 650 msnm; en el costado oriental de la Cordillera Oriental, en los municipios de Casanare, alcanza altitudes que van desde los 1.350 a los 1.525 msnm, y, por último, en algunos municipios de Boyacá y Santander, sobre la Cordillera Oriental, se la encuentra desde los 1.500 hasta los 2.400 msnm (34).

Las poblaciones de la región occidental del país parecen no poseer capacidad de colonización, pues no presentan ningún grado de domiciliación, ya que los pocos individuos reportados en

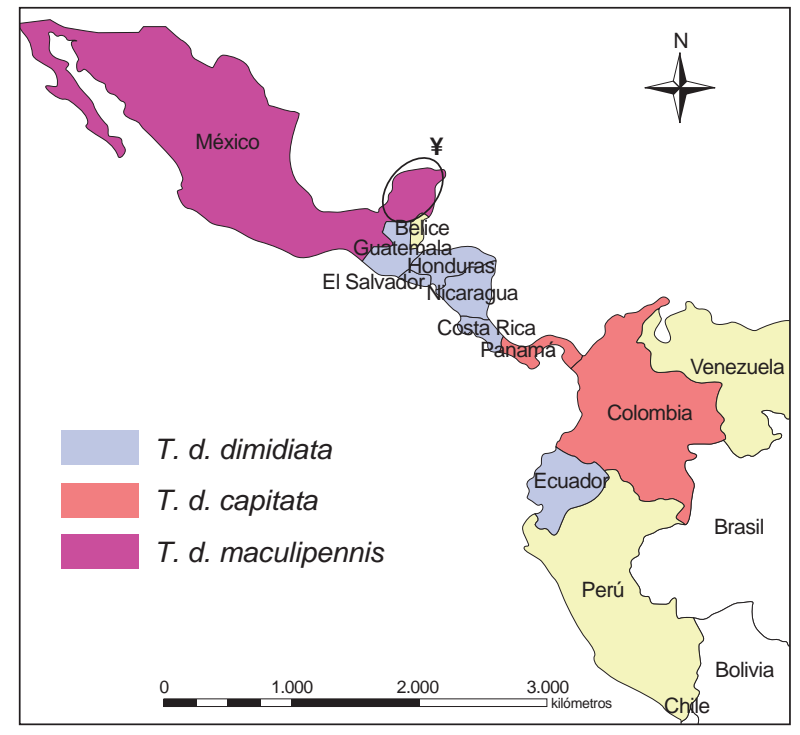

Figura 1. Distribución de Triatoma dimidiata en América Latina. $¥$ Zona de hibridación entre $T$. d. maculipennis y $T$. sp. affin a dimidiata

viviendas han sido adultos, al parecer, atraídos por la luz artificial (Parra-Henao G, Angulo V, Jaramillo N, Restrepo M. Factores de riesgo de infestación domiciliaria por Triatoma dimidiata. Biomédica. 2009;29(supl.1):316. Memorias, XIV Congreso Colombiano de Parasitología y Medicina Tropical y $X$ Simposio PECET). En Antioquia, se ha reportado la presencia de algunos ejemplares adultos en domicilios y peridomicilios en cuatro municipios de la zona de Urabá (Apartadó, Chigorodó, Necoclí y Turbo) (31). Al parecer, dichos insectos eran de origen silvestre y llegaron a estos hábitats de manera accidental, sin que se establecieran en las casas, por lo que su presencia correspondía a intrusiones desde el hábitat selvático sin que se diera la colonización. En los departamentos de Magdalena, Bolívar y Sucre (incluida la Sierra Nevada de Santa Marta), se ha podido determinar que el hábitat de esta especie es silvestre y se ha asociado principalmente a las palmas de la especie Attalea butyracea, aunque se han constatado algunas intrusiones en las viviendas (35). Debido a esta característica, el involucramiento vectorial de $T$. dimidiata en la región occidental es poco, con hábitos estrictamente silvestres asociados con las palmas (36).

Por el contrario, se ha reportado que las poblaciones de la región centro-oriental habitan en el domicilio y el peridomicilio, particularmente en los departamentos de Boyacá y Santander (7), incluso, en viviendas en buenas condiciones de construcción 
ubicadas en las cabeceras municipales (37). En esta región se han evidenciado focos silvestres desde los cuales se dan intrusiones y colonización de las viviendas. En algunos estudios de campo en el municipio de Boavita, se ha registrado la presencia de poblaciones silvestres de $T$. dimidiata en cuevas, las cuales constantemente incursionan en los ambientes domésticos y peridomésticos (38), dinámica que ha exigido una estricta vigilancia entomológica posterior al tratamiento con insecticidas en esos dos departamentos, en donde se han observado ninfas y adultos de $T$. dimidiata en las viviendas y en las estructuras peridomiciliarias a pesar del control químico, probablemente debido a dichas intrusiones $(39,40)$.

Esta capacidad de movilización también se ha constatado en los estudios genéticos; al analizar la subunidad 4 (ND4) de la NADH deshidrogenasa, Grisales, et al., detectaron diferenciación genética (Hd: 0,863) y estructuración entre poblaciones de T. dimidiata $\left(\phi_{\mathrm{ST}}: 0,761\right)$ de los departamentos de La Guajira y Cesar (región Caribe) y Santander (región Andina), lo cual sugiere que la gran variabilidad genética de la población en Santander podría estar asociada con las diferencias ecológicas observadas en ese departamento, en donde los individuos silvestres y peridomiciliarios tienden a la domiciliación. Por el contrario, la baja variabilidad genética de la población de $T$. dimidiata en La Guajira podría relacionarse con el estricto hábitat arbóreo que ocupa en la región (41).

Mediante análisis moleculares de la citocromo oxidasa I (COI) y de multilocus de microsatélites (Multilocus Microsatellite Analysis, MMA) en ejemplares de $T$. dimidiata de una región más amplia de Colombia (seis departamentos de las regiones Caribe y Andina: Santander, Boyacá, Norte de Santander, Bolívar, Cesar y Magdalena), GómezPalacio, et al., evidenciaron la presencia de tres grupos de genes correspondientes a tres regiones biogeográficas del país: llanuras del Caribe, Sierra Nevada de Santa Marta y valles interandinos. Además, se encontró un grupo que se diferenciaba de estas tres ecorregiones, lo cual sugería un posible flujo de genes entre las regiones, o la presencia de haplótipos de un origen antiguo (42).

Estos hallazgos, así como el conocimiento actual sobre los hábitats naturales y artificiales y el proceso de dispersión de esta especie, permiten sugerir que en Colombia existen dos poblaciones: una que habita fundamentalmente en palmas, con pocos eventos de colonización en las viviendas, es decir, de bajo riesgo epidemiológico, en el occidente, y la otra, que se encuentra en cuevas y formaciones rocosas y que, al parecer, es la fuente de las poblaciones domiciliares implicadas en la transmisión de $T$. cruzi en el centro-oriente del país (valles interandinos) (figura 2).

En el cuadro 1 se resume la información existente sobre la ubicación geográfica de $T$. dimidiata, su ecología, domesticidad e infección con T. cruzi, así como su seroprevalencia en humanos en las zonas de estudio.

\section{Estatus taxonómico}

Antes se hizo referencia al posible origen de $T$. dimidiata y su dispersión desde la península de Yucatán, lo cual dio origen a variaciones morfológicas clasificadas como subespecies por Usinger en 1944, a partir de sus trabajos sobre las diferencias en las proporciones de la cabeza, los ojos y la forma de los húmeros ( $T$. d. capitata, $T$. $d$. maculipennis y T. d. dimidiata) (24). En 1979, Lent, et al., eliminaron las categorías de subespecies e interpretaron estas variantes con base en un efecto clonal y propusieron la división del género Triatoma en dos grupos principales: el grupo Protracta y el

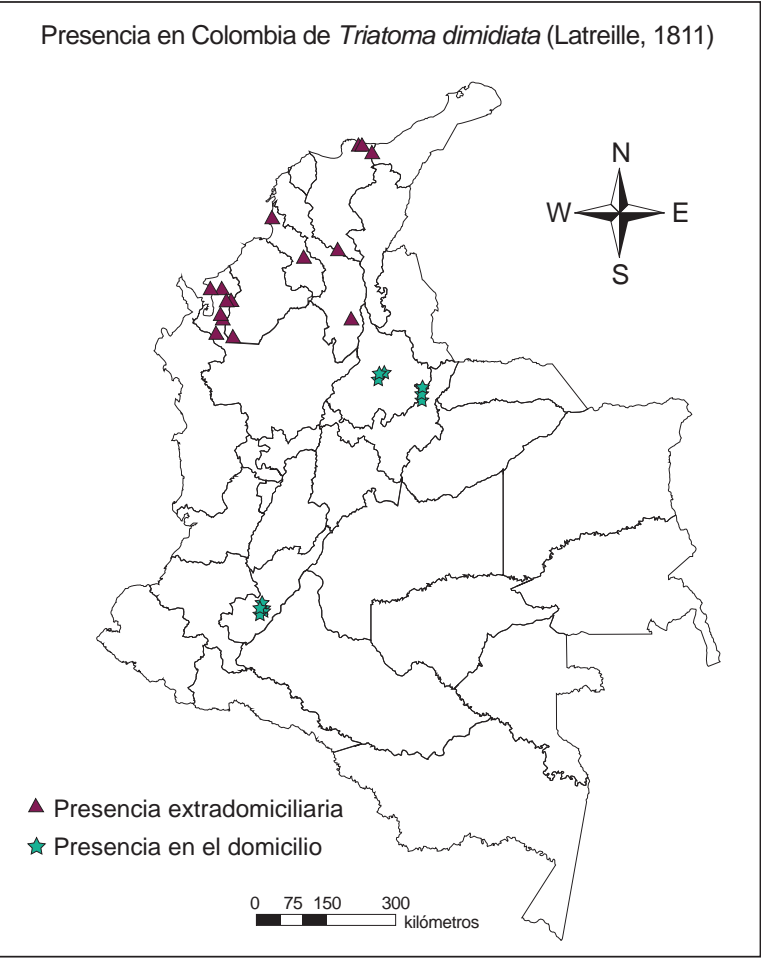

Figura 2. Distribución geográfica de las dos poblaciones de Triatoma dimidiata en Colombia: presencia extradomiciliaria (silvestre) en el occidente, y en el domicilio en la zona centrooriental 
Cuadro 1. Matriz de resumen de los datos sobre aspectos entomológicos, epidemiológicos, ecológicos y de distribución de Triatoma dimidiata en Colombia

\begin{tabular}{|c|c|c|c|c|c|c|}
\hline Departamento & $\begin{array}{l}\text { Número de } \\
\text { municipios }\end{array}$ & $\begin{array}{l}\text { Número } \\
\text { de veredas }\end{array}$ & $\begin{array}{l}\text { Zonas de vida } \\
\text { (Holdridge) }\end{array}$ & $\begin{array}{l}\text { Colonización } \\
\text { de viviendas }\end{array}$ & $\begin{array}{l}\text { Presencia } \\
\text { de } T \text {. cruzi }\end{array}$ & $\begin{array}{l}\text { Seroprevalencia } \\
\text { en humanos (\%) }\end{array}$ \\
\hline $\begin{array}{l}\text { Antioquia } \\
\text { Arauca }\end{array}$ & $\begin{array}{l}4 \\
1\end{array}$ & $\begin{array}{l}4 \\
1\end{array}$ & $\begin{array}{c}\text { BHT, BMHT } \\
\text { BST }\end{array}$ & $\begin{array}{l}\text { No } \\
\text { No }\end{array}$ & $\begin{array}{l}\text { Sí } \\
\text { No }\end{array}$ & $\begin{array}{l}2,1 \\
2,9\end{array}$ \\
\hline Boyacá & 21 & 91 & $\begin{array}{c}\text { BMHPM, BST,BHMB, } \\
\text { BMHMB, BHT }\end{array}$ & Sí & Sí & 5,6 \\
\hline Casanare & 2 & 2 & BSMB & Sí & Sí & 7,84 \\
\hline Cesar & 10 & 19 & BST, BHT & Sí & No & -- \\
\hline Cundinamarca & 1 & & BHMB & Sí & Sí & 5 \\
\hline La Guajira & 1 & 1 & BST & No & No & -- \\
\hline Huila & 1 & & BST & No & No & -- \\
\hline Magdalena & 4 & 8 & BST, BHT & No & Sí & 0,32 \\
\hline Meta & 2 & & BMHT & Sí & No & 1,78 \\
\hline Norte de Santander & 2 & 1 & BHPM, BHT & Sí & Sí & 2,6 \\
\hline Santander & 29 & 72 & $\begin{array}{c}\text { BMHMB, BHT, BMHT, } \\
\text { BSMB }\end{array}$ & Sí & Sí & 1,74 \\
\hline San Andrés & 1 & 1 & BST & No & No & -- \\
\hline Sucre & 1 & & BST & No & Sí & 0,032 \\
\hline
\end{tabular}

BHT: bosque húmedo tropical; BMHT: bosque muy húmedo tropical; BST: bosque seco tropical; BSMB: bosque seco montano bajo; BMHPM: bosque muy húmedo premontano; BHMB: bosque húmedo montano bajo; BHPM: bosque húmedo premontano; BMHMB: bosque muy húmedo montano bajo

grupo Rubrofasciata (43). En el 2002, Dujardin, et al., modificaron la clasificación inicial del género e incluyeron nueve complejos y seis subcomplejos correspondientes a áreas geográficas diferentes (44) (cuadro 2).

\section{Caracterización genotípica y fenotípica}

En los estudios moleculares se han diseñado marcadores para tipificar y caracterizar la dinámica poblacional de T. dimidiata. En el 2002, Anderson, et al., describieron ocho loci polimorfos de secuencias cortas repetidas (microsatélites) para la especie (45). Asimismo, se han hecho análisis con otros marcadores nucleares mediante amplificación aleatoria de ADN polimorfo (Random Amplification of Polymorphic DNA, RAPD) $(38,46)$, y marcadores mitocondriales como una fracción del gen de la subunidad 16s -mtlsurRNA y del citocromo b -mtCytB (47).

Quizá uno de los marcadores más informativos hasta ahora para la descripción de la estructura poblacional de esta especie es el segundo espaciador intergénico ribosómico nuclear (Internal Transcribed Spacer, ITS-2). Este marcador ha permitido establecer importantes variaciones puntuales en nucléotidos y en el número de secuencias cortas repetidas (microsatélites) entre diferentes poblaciones de México, Honduras, Nicaragua y Ecuador $(20,48)$. Al comparar la parsimonia y las secuencias del ITS-2 de varias especies de las tribus Triatomini y Rhodniini, Marcilla, et al., observaron muy poca variación entre las especies pertenecientes al complejo Phyllosoma, con ausencia total de diferencias en los nucléotidos, a excepción de $T$. dimidiata, que presenta un nucleótido diferente (20). Por lo tanto, se considera que el grupo de especies del complejo es monofilético, lo cual sugiere un ancestro común. Estos trabajos han revelado una compleja estructura poblacional de $T$. dimidiata relacionada con aspectos epidemiológicos importantes, como la capacidad de realizar con éxito un eventual proceso de transición de hábitats, altas tasas de infección natural y áreas biogeográficas con mayor riesgo potencial $(21,38)$.

Por su parte, Panzera, et al., reportaron variaciones en el tamaño del genoma y diferencias en el citotipo de poblaciones de México, Guatemala, El Salvador y Colombia. Se encontraron tres citotipos, de los cuales el número 1 se halló en las poblaciones de los cuatro países asociado con ambientes domésticos, peridomésticos y selváticos, en tanto que los citotipos 2 y 3 se asociaron a poblaciones selváticas, lo cual sugiere que se trata de especies crípticas correspondientes a los tres citotipos con diferente riesgo epidemiológico para la enfermedad de Chagas (21). Por el contrario, mediante análisis del ITS-2 y su correspondencia filogenética, Bargues, et al., sugirieron reasignar el estatus subespecífico de algunos grupos biogeográficos, aunque no había información suficiente que sustentara la elevación del rango a nivel específico; en ese marco, $T$. dimidiata capitata fue la única subespecie asignada para el país (22). Según el estudio de estos autores, se reubicaron las subespecies identificadas y, además, se describió una 
Cuadro 2. Triatoma dimidiata según la clasificación propuesta por Dujardin, et al., 2002

\begin{tabular}{ll}
\hline Grupo Proctata & Grupo Rubrofasciata \\
\hline Complejo Lecticularia & Subgrupo Infestans \\
indictiva, lecticularia, sanguisuga & Tres complejos, seis subcomplejos, 34 especies \\
Complejo Proctacta & Subgrupo Rubrofasciata \\
barberi, incrassata, neotomae, nitida, peninsularis, & Cuatro complejos, 27 especies \\
proctacta, sinaloensis & Triatoma dimidiata hace parte del complejo Phyllosoma \\
\hline
\end{tabular}

nueva especie, T. spp. affinis dimidiata, y se integró una especie insular, la $T$. hegneri, al complejo con el nuevo nombre de T.d. hegneri. En conclusión, el estado taxonómico actual de $T$. dimidiata reconoce dos especies: T. spp. affinis dimidiata y T. dimidiata, y cuatro subespecies: T. d. hegneri, T. d. dimidiata, $T$. d. maculipennis y $T$. d. capitata, lo cual permite su reconocimiento como complejo (49).

Zeledón, et al., Jurberg, et al., y Schofield han considerado que $T$. dimidiata es un ensamblaje de poblaciones morfológicas de gran variedad y han planteado la existencia de un complejo de especies con base en las variaciones de tamaño y coloración $(29,50,51)$. No obstante, en términos generales, se trata de una especie con individuos de tamaño grande y colorido distintivo en el cuerpo: de píceo a negro en el conexivo y de amarillo pálido a amarillo naranja en el corium (hemelytron). El macho mide entre 24,5 y $32 \mathrm{~mm}$ y, la hembra, entre 24,5 y 35 $\mathrm{mm}(43,50)$ (figura 3$)$. La variación morfológica entre las poblaciones de triatominos podría estar asociada con la ecogeografía, la preferencia de huésped y el hábitat (52-54). En el caso de $T$. dimidiata, en algunos estudios se ha logrado discriminar y correlacionar las poblaciones con su procedencia geográfica, lo que, al parecer, es una de las principales causas de las divergencias evolutivas $(55,56)$.

En estudios comparativos de los patrones sensoriales de las antenas de $T$. dimidiata en Colombia, se han señalado variaciones en la densidad de las sensilias asociadas con el hábitat (57); igualmente, se ha observado variación cromática en especímenes recolectados en diferentes regiones del país (Parra-Henao, observación personal). En su estudio sobre cuatro poblaciones de $T$. dimidiata en Colombia, Esteban-Adarme hizo la descripción morfológica de caracteres métricos de la cabeza, la morfología de la genitales externos y los patrones de coloración, y halló que las poblaciones del occidente (departamentos de Cesar y Magdalena) presentaban mayores tamaños de la cabeza, los ojos y el ocelo que las demás poblaciones estudiadas, así como un mayor número de dentículos del proceso del endosoma; describió, asimismo, un patrón de coloración uniformemente negro en el pronoto, característico de las poblaciones en el oriente (departamentos de Boyacá y Santander), en tanto que en el occidente encontró que el lóbulo posterior era negro con líneas laterales amarillas y anaranjadas. Las características descritas en dicho estudio sugerían la existencia de dos grupos morfológicos distinguibles, cuya diferenciación podría deberse a factores geográficos o ecológicos (53).

\section{Comportamiento y alimentación}

Triatoma dimidiata presenta un comportamiento ecléctico, con un amplio espectro de huéspedes, los cuales incluyen desde especies silvestres y domésticas hasta el hombre. La sangre humana es la más comúnmente hallada en los insectos recolectados en el domicilio y peridomicilio (34). La especie ocupa ecótopos tanto naturales como artificiales, con éxito parcial o completo. Si las condiciones del refugio lo permiten, los perros y las gallinas son sus huéspedes más frecuentes, aunque un factor importante en la visita de los adultos silvestres a los domicilios humanos es la atracción hacia la luz artificial (58). El hallazgo de sangre de reservorios silvestres en el contenido estomacal de $T$. dimidiata, asociado con las altas tasas de infección natural, indicaría que participa de manera activa en el transporte de $T$.

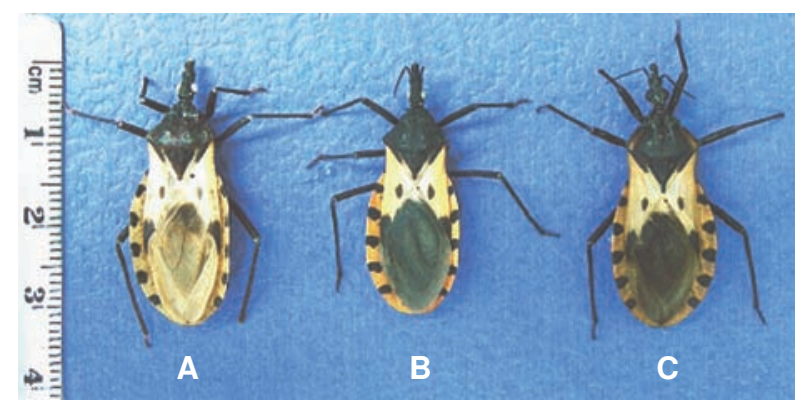

Figura 3. Variación cromática en especímenes de Triatoma dimidiata procedentes de diferentes regiones de Colombia. A. Huila. B. Boyacá. C. Sierra Nevada de Santa Marta. Fotografía: Gabriel Parra-Henao 
cruzi hacia el ambiente doméstico, y que es una fuente importante de infestación y reinfestación de construcciones humanas (59-61).

La capacidad de $T$. dimidiata para reinfestar las viviendas ha llevado a estudiar esta característica después de la aspersión química; en este sentido, Dumontiel, et al., hicieron un estudio piloto en Yucatán para evaluar la infestación antes y después de la aspersión con ciflutrina (Solfac ${ }^{\circledR}$, Bayer) en el domicilio, el peridomicilio y los hábitats selváticos. Durante el estudio, se encontraron especímenes vivos en el domicilio en los primeros cuatro meses después de la aspersión. Los vectores empezaron a visitar los domicilios a partir de marzo y hasta finales de junio. Se determinó que, en los domicilios, predominaron los especímenes adultos, en tanto que los estadios de ninfa se recolectaron en el peridomicilio y en hábitats selváticos, lo cual sugiere que los adultos migraron de sitios cercanos y que su presencia no se debía a su supervivencia a la aspersión (62).

Torres-Montero, et al., determinaron la infestación y las fuentes de alimentación de $T$. dimidiata en viviendas ubicadas en el centro del estado de Veracruz, México. Mediante amplificación del gen mitocondrial citocromo b, se identificó la presencia de sangre humana en el $87,5 \%$ de los triatominos del domicilio, seguida de sangre de ratón en $25 \%$ y de rata en $20 \%$ y, en menor medida, de sangre de gallina, gato y perro. Por el contrario, en los individuos capturados en el peridomicilio, predominó la sangre de rata (50\%), seguida de la de humano (25\%). Se encontró una triple infección por sangre humana, de gallina y de caballo, lo cual corrobora la movilidad de $T$. dimidiata entre el domicilio y el peridomicilio (63).

En Colombia, Farfán-García, et al., mediante ELISA, detectaron proteínas sanguíneas en el contenido intestinal de individuos de $T$. dimidiata capturados en el domicilio y fuera de él en los municipios de Capitanejo y Macaravita en el departamento de Santander. En general, la sangre de animales domésticos fue la más frecuente $(40,6 \%)$, seguida de la sangre de huéspedes silvestres y humanos (4,6 \% y $4,7 \%$, respectivamente). En $43,1 \%$ de los individuos capturados en el domicilio se encontró sangre de gallina, sangre humana en $31,4 \%$ de ellos, de perro en $17,6 \%$ y de caprino en $15,7 \%$. En los especímenes provenientes del peridomicilio y del hábitat silvestre, no se detectó sangre humana, siendo los animales domésticos y el armadillo las fuentes de preferencia. Es importante destacar el hallazgo de sangre de animales silvestres, como la zarigüeya y el armadillo, en 7,8 \% de los insectos capturados en las viviendas, al igual que sangre de animales domésticos en los especímenes del hábitat silvestre, lo cual evidencia, una vez más, la movilidad de $T$. dimidiata (12).

En condiciones experimentales, se ha evaluado el tiempo de alimentación en conejos y de defecación de colonias de $T$. dimidiata mantenidas en condiciones de humedad relativa de $65 \pm 10 \%$ y de temperatura de $27,3 \pm 3{ }^{\circ} \mathrm{C}$. No se observaron diferencias en el tiempo de alimentación y el transcurrido hasta la defecación en los estadios de ninfa. El primer estadio de ninfa fue el que más tiempo empleó en su alimentación $(25 \pm 2$ minutos) y el cuarto estadio el que menos tiempo empleó (15 \pm minutos). Un comportamiento igual se determinó en cuanto al tiempo transcurrido entre la alimentación y la defecación ( $45 \pm 6$ minutos $V s .21$ \pm 8 minutos, respectivamente). Hembras y machos necesitaron el mismo tiempo para alimentarse (15 \pm 5 minutos $V s$. $14 \pm 5$ minutos), y también fue el mismo el transcurrido hasta la defecación (20 \pm 6 minutos Vs. $21 \pm 7$ minutos) (64).

\section{Factores asociados con la presencia de triatominos en viviendas humanas}

La enfermedad de Chagas ocurre en contextos caracterizados por determinados factores socioculturales, políticos, económicos, ambientales e históricos directamente relacionados con la dispersión de los triatominos (65). Las condiciones socioeconómicas son determinantes para la infestación domiciliaria y, en algunos estudios, se ha reportado que la probabilidad de encontrar individuos de $T$. dimidiata es 4,3 a 10 veces mayor en viviendas con condiciones socioeconómicas deficientes que en aquellas con buenas condiciones (17).

Algunas de las características de la vivienda asociadas con la presencia de $T$. dimidiata incluyen las paredes no revocadas o de revocado incompleto (odds ratio, OR=3,9; $\mathrm{IC}_{95 \%} 1,9-7,7$ ), las paredes de bahareque $\left(\mathrm{OR}=4,8 ; \mathrm{IC}_{95 \%}, 1,4-17,1\right) \quad(8,66)$, las paredes de adobe comparadas con las de bloque (OR=9,55; $\left.\mathrm{IC}_{95 \%} 2,1-44,5\right)$ (17), el piso de tierra (OR=10,1; IC $\left.\mathrm{I}_{95 \%} 5,3-18,04\right)$, tener tres o más habitaciones $\left(\mathrm{OR}=2,04 ; \mathrm{IC}_{95 \%}, 1,1-3,9\right)$, los techos de lámina de cartón (OR=6,8; $\left.\mathrm{IC}_{95 \%}, 1,5-31,3\right)(67)$, los techos mixtos (teja y palma) (OR=36,1; $I C_{95 \%}$ $12,2-106,9)$, la presencia de gatos $(\mathrm{OR}=3,94$; $\left.\mathrm{IC}_{95 \%} 1,4-11\right)$, y la acumulación de rocas (OR=5,3; $\mathrm{IC}_{95 \%}$ 1,6-17) (8). También, se ha reportado como factor asociado con la infestación la localización 
de la casa cerca de la periferia de la localidad, la presencia de gallineros y de dos o más perros $(8,67)$. No obstante, Zeledón, et al., reportaron la colonización por especímenes silvestres de $T$. dimidiata de un ecótopo artificial en el patio trasero de una casa nueva, bien construida, en Santa Ana, provincia de San José, en Costa Rica (61).

Otro de los factores asociados con la infestación domiciliaria ha sido la luz artificial. PachecoTucuch, et al., realizaron un estudio en las villas rurales de Bokoba, Teya y Sudzal, en la parte central del estado de Yucatán, México, en el cual evaluaron la relación entre la infestación de las casas y las fuentes de luz, y la compararon con la distancia entre la casa y dichas fuentes. El análisis cuantitativo mostró una relación inconsistente entre las luces de la parte externa de las viviendas y las viviendas infestadas; solo en villa Boboka se encontró una relación significativa $(p=0,028, O R$; $\mathrm{IC}_{95 \%}$ 1,2-2,2). Los autores encontraron, en cambio, una asociación estadística entre las viviendas infestadas y las luces públicas $(p=0,008)$. Las casas infestadas estaban a $18 \pm 0,6 \mathrm{~m}$ de la luz pública $y$, las no infestadas, a $22,6 \pm 0,4 m(p<0,001)$. Un análisis experimental adicional evidenció que tanto machos como hembras adultas de $T$. dimidiata se vieron fuertemente atraídos a túneles con luz blanca $(p<0,001)(58)$.

Las condiciones climáticas también inciden en el número de insectos capturados durante el año, el cual aumenta en las temporadas secas, que en Centroamérica corresponden a los meses de marzo y septiembre $(68,69)$. En algunos estudios, se ha examinado la relación entre las variables climáticas y la presencia de $T$. dimidiata utilizando sistemas de información geográfica y los parámetros registrados en las estaciones. Bustamante, et al., por ejemplo, encontraron que la temperatura absoluta máxima, es decir, la temperatura más alta registrada en un año, se asoció significativamente con la presencia de $T$. dimidiata (70) y, mediante modelos de nicho ecológico, Parra-Henao, et al., encontraron una asociación con el índice de vegetación, la temperatura estacional y la precipitación anual en poblaciones recolectadas en las regiones centrooriental y noroccidental del país (71).

La distribución y el número de especímenes pueden variar considerablemente ante la presencia de fenómenos naturales, como huracanes o inundaciones. Guzmán-Tapia, et al., estudiaron los cambios en la distribución de $T$. dimidiata después del paso del huracán Isidoro, que afectó la costa norte del golfo de México. Los autores recolectaron especímenes en enero de 2003, tres meses después del huracán, y hasta diciembre de ese año. El principal hallazgo reportado fue la asociación del paso del huracán con las variaciones a mediano y largo plazo en la infestación domiciliaria por $T$. dimidiata. Se observó un aumento en el número de insectos adultos en el trimestre de enero a marzo, comparado con el mismo periodo en años anteriores $(p=0,04)$, lo cual sugería que se habían producido nuevas llegadas de insectos (72).

\section{Conclusiones}

El comportamiento ecléctico de $T$. dimidiata en el contexto de una economía y una situación geopolítica inestables y en ausencia de la supresión permanente del vector, plantea un complejo panorama de riesgo epidemiológico, lo cual se suma al incremento de procesos poco comunes en la transmisión de $T$. cruzi, como han sido los recientes brotes de infección por vía oral (73-76). La situación de $T$. dimidiata en Colombia requiere que los investigadores y estudiosos propongan estrategias novedosas para el control de los vectores.

Como alternativa al control con insecticidas, se han modificado las viviendas teniendo en cuenta los factores asociados con la presencia domiciliaria de $T$. dimidiata. Dichas modificaciones han incluido el cambio de elementos estructurales de deficiente calidad por materiales suaves y planos que no se conviertan en potenciales refugios de los insectos. Algunas de estas estrategias han sido exitosas y han logrado reducir las tasas de infestación hasta en $96,4 \%$, en tanto que las intervenciones combinadas de aspersión con insecticidas piretroides y modificaciones en las viviendas han logrado reducciones de hasta $100 \%$ (77).

Las intervenciones integrales se han basado en el concepto de "ecosalud", el cual tiene en cuenta los factores ecológicos y sociales determinantes de la transmisión del parásito, y el trabajo intersectorial y educativo en la comunidad, con el fin de garantizar la sostenibilidad de las intervenciones (78). Este tipo de estrategias concita una gran participación de la comunidad y permite que las medidas perduren, tanto en lo referente a la estructura de las viviendas (79) como al control de animales asociados con la infestación de vectores (80). Por ello, su importancia ha sido reconocida por la OMS, ya que tienen en cuenta las realidades sociales y culturales de las poblaciones afectadas e incorporan de manera integral sus intereses y necesidades en los sistemas de vigilancia y control 
(81). En tales estrategias, se ha recurrido a diversas metodologías cualitativas que incluyen entrevistas individuales y grupales, etnografía y observación $(65,82)$.

La Red Chagas Colombia ha venido estudiando los factores socioculturales determinantes y las dinámicas de acceso al diagnóstico y a la atención integral en algunos departamentos del país, con el fin de comprender los significados culturales de la enfermedad para incorporarlos a las estrategias de prevención, control y tratamiento integral (83).

Estas aproximaciones cualitativas y mixtas son de gran utilidad, por lo cual el llamado es a continuar trabajando desde esta perspectiva y con un enfoque más específico en torno a los aspectos culturales del riesgo, de la enfermedad de Chagas, los vectores y el significado de la prevención. Ello podría tener efectos futuros positivos al incentivar una mejor comprensión, apropiación y empoderamiento de las comunidades en torno a la dinámica de la transmisión de la enfermedad por la vía vectorial y, especialmente, a su prevención, así como al mejoramiento de las condiciones habitacionales y la promoción de condiciones saludables en el domicilio y el peridomicilio.

\section{Conflicto de intereses}

Los autores declaramos que no existieron conflictos de intereses con respecto a la concepción, desarrollo y la publicación de este trabajo.

\section{Financiación}

Red Chagas Colombia, contrato Colciencias 3802011, código 5014-537-30398, y por la convocatoria 567 de Colciencias para becas de doctorado a nivel nacional.

\section{Referencias}

1. Carod-Artal FJ, Gascon J. Chagas disease and stroke. Lancet Neurol. 2010;9:533-42. http://dx.doi.org/10.1016/ S1474-4422(10)70042-9

2. Pereira Nunes MC, Dones W, Morillo CA, Encina JJ, Ribeiro AL. Council on Chagas Disease of the Interamerican Society of Cardiology. Chagas disease: An overview of clinical and epidemiological aspects. J Am Coll Cardiol. 2013;62:767-76. http://dx.doi.org/10.1016/j.jacc. 2013.05.046

3. World Health Organization. Chagas disease in Latin America: An epidemiological update based on 2010 estimates. Wkly Epidemiol Rec. 2015;90:33-43.

4. Araujo LM, Ferreira MLB, Nascimento OJ. GuillainBarré syndrome associated with the Zika virus outbreak in Brazil. Arq Neuropsiquiatr. 2016;74: 253-5. http://dx.doi. org/10.1590/0004-282X20160035
5. Watrin L, Ghawché F, Larre P, Neau J-P, Mathis S, Fournier E. Guillain-Barré syndrome (42 cases) occurring during a Zika virus outbreak in French Polynesia. Medicine (Baltimore). 2016;95:e3257. http://dx.doi.org/10.1097/MD. 0000000000003257

6. de Oliveira CS, da Costa Vasconcelos PF. Microcephaly and Zika virus. J Pediatr (Rio J). 2016;92:103-5. http:// dx.doi.org/10.1016/j.jped.2016.02.003

7. Guhl F, Aguilera G, Pinto N, Vergara D. Updated geographical distribution and ecoepidemiology of the triatomine fauna (Reduviidae: Triatominae) in Colombia. Biomédica. 2007;27(Supl.1):143-62. http://dx.doi.org/10. 7705/biomedica.v27i1.258

8. Parra-Henao G, Cardona ÁS, Quirós-Gómez O, Angulo V, Alexander $\mathbf{N}$. House-level risk factors for Triatoma dimidiata infestation in Colombia. Am J Trop Med Hyg. 2015;92:193-200. http://dx.doi.org/10.4269/ajtmh.14-0273

9. Gómez-Palacio A, Triana $\mathrm{O}$, Jaramillo-O N, Dotson EM, Marcet PL. Eco-geographical differentiation among Colombian populations of the Chagas disease vector Triatoma dimidiata (Hemiptera: Reduviidae). Infect Genet Evol J. 2013;20:352-61. http://dx.doi.org/10.1016/j.meegid. 2013.09.003

10. Guhl F, Pinto N, Aguilera G. Sylvatic triatominae: A new challenge in vector control transmission. Mem Inst Oswaldo Cruz. 2009;104(Suppl.1):71-5. http://dx.doi.org/10.1590/ S0074-02762009000900012

11. Reyes M, Angulo VM, Sandoval CM. Efecto tóxico de b-cipermetrina, deltametrina y fenitrotión en cepas de Triatoma dimidiata (Latreille, 1811) y Triatoma maculata (Erichson, 1848) (Hemiptera, Reduviidae). Biomédica. 2007;27(Supl.1):75-82. http://dx.doi.org/10.7705/biomedica. v27i1.250

12. Farfán-García AE, Angulo-Silva VM. Triatoma dimidiata populations (Hemiptera: Reduviidae: Triatominae) feeding behaviour in an endemic zone and related epidemiological implications. Rev Salud Pública. 2011;13:163-72. http:// dx.doi.org/10.1590/S0124-00642011000100014

13. Chaverra-Rodríguez D. Morfología y taxonomía de triatominos. En: Fronteras de investigación en enfermedades infecciosas: modelo enfermedad de Chagas. Primera edición. Medellín: Universidad de Antioquia; 2011. p. 23-44.

14. Monroy MC, Bustamante DM, Rodas AG, Enríquez ME, Rosales RG. Habitats, dispersion and invasion of sylvatic Triatoma dimidiata (Hemiptera: Reduviidae: Triatominae) in Petén, Guatemala. J Med Entomol. 2003;40:800-6. http:// dx.doi.org/10.1603/0022-2585-40.6.800

15. Zeledón R, Calvo N, Montenegro VM, Lorosa ES, Arévalo C. A survey on Triatoma dimidiata in an urban area of the province of Heredia, Costa Rica. Mem Inst Oswaldo Cruz. 2005;100:507-12. http://dx.doi.org/S007402762005000600002

16. Guzmán-Tapia Y, Ramírez-Sierra MJ, Dumonteil E. Urban infestation by Triatoma dimidiata in the city of Mérida, Yucatán, México. Vector Borne Zoonotic Dis. 2007;7:597606. http://dx.doi.org/10.1089/vbz.2007.0133

17. Bustamante DM, Monroy C, Pineda S, Rodas A, Castro $\mathrm{X}$, Ayala V, et al. Risk factors for intradomiciliary infestation by the Chagas disease vector Triatoma dimidiata in Jutiapa, Guatemala. Cad Saúde Pública. 2009;25(Suppl.1):S83-92. http://dx.doi.org/10.1590/S0102-311X2009001300008 
18. World Health Organization. Control of Chagas disease: Second report of the WHO expert committee. Geneva: World Health Organization; 2002.

19. Dorn PL, Monroy C, Curtis A. Triatoma dimidiata (Latreille, 1811): A review of its diversity across its geographic range and the relationship among populations. Infect Genet Evol. 2007;7:343-52. http://dx.doi.org/10.1016/j.meegid. 2006.10.001

20. Marcilla A, Bargues MD, Ramsey JM, Magallón-Gastelum E, Salazar-Schettino PM, Abad-Franch F, et al. The ITS-2 of the nuclear rDNA as a molecular marker for populations, species, and phylogenetic relationships in Triatominae (Hemiptera: Reduviidae), vectors of Chagas disease. Mol Phylogenet Evol. 2001;18:136-42. http://dx.doi.org/10.1006/ mpev.2000.0864

21. Panzera F, Ferrandis I, Ramsey J, Ordóñez R, SalazarSchettino PM, Cabrera M, et al. Chromosomal variation and genome size support existence of cryptic species of Triatoma dimidiata with different epidemiological importance as Chagas disease vectors. Trop Med Int Health. 2006;1:1092103. http://dx.doi.org/10.1111/j.1365-3156.2006.01656.x

22. Bargues MD, Klisiowicz DR, González-Candelas F, Ramsey JM, Monroy C, Ponce C, et al. Phylogeography and genetic variation of Triatoma dimidiata, the main Chagas disease vector in Central America, and its position within the genus Triatoma. PLoS Negl Trop Dis. 2008;2:e233. http:// dx.doi.org/10.1371/journal.pntd.0000233

23. Herrera-Aguilar M, Be-Barragán LA, Ramírez-Sierra MJ, Tripet F, Dorn P, Dumonteil E. Identification of a large hybrid zone between sympatric sibling species of Triatoma dimidiata in the Yucatán peninsula, México, and its epidemiological importance. Infect Genet Evol J. 2009;9:1345-51. http://dx. doi.org/10.1016/j.meegid.2009.09.009

24. Usinger RL. The triatominae of North and Central America and the West Indies and their public health significance. Washington, D.C.: Government Printing Office; 1944.

25. Otálora B. Triatoma dimidiata (Latreille). An Soc Biol. 1952;5:135-7.

26. Ucrós H, Rocha H, Duque M. Distribución de Triatominae en Colombia. Antioquia Médica. 1971;8:707-17.

27. Marinkelle $\mathbf{C}$. The distribution of Colombian Triatominae and their infestation with trypanosomatid flagellates. Geneve: World Health Organization; 1969.

28. D'Alessandro A, Barreto P, Thomas M. Nuevos registros de triatominos domiciliarios y extradomiciliarios en Colombia. Colomb Med. 1981;12:75-85

29. Zeledón R, Rabinovich JE. Chagas disease: An ecological appraisal with special emphasis on its insect vectors. Annu Rev Entomol. 1981;26:101-33. http://dx.doi.org/10.1146/ annurev.en.26.010181.000533

30. Corredor A, Santacruz M, Páez S, Guateme L. Distribución de los triatominos domiciliarios en Colombia. Bogotá: Instituto Nacional de Salud; 1990.

31. Restrepo M, Restrepo C, Parra G, Salazar C. Estudios sobre la tripanosomiasis americana en el Instituto Colombiano de Medicina Tropical. En: Curso Taller Internacional-Sistemas de información geográfica, sensores remotos y genética poblacional de vectores y parásitos aplicados al control de la enfermedad de Chagas. Bogotá: Universidad de los Andes; 2002.
32. Parra-Henao G, Angulo V, Jaramillo N, Restrepo M. Triatominos (Hemiptera: Reduviidae) de la Sierra Nevada de Santa Marta, Colombia. Aspectos epidemiológicos, entomológicos y de distribución. CES Med. 2010;23:17-26.

33. Parra-Henao G, Flórez-Martínez M, Angulo Silva V. Vigilancia de Triatominae (Hemiptera: Reduviidae) en Colombia. Primera edición. Bogotá: Sic Editorial Ltda; 2015. p. 127.

34. Angulo V. Triatoma dimidiata en Colombia, aspectos biológicos y eco-epidemiológicos. Curso Taller Internacional: el uso de los sistemas de información geográfica y sensores remotos (SR) en salud pública. Bogotá: Universidad de los Andes; 2006.

35. Parra-Henao G, Restrepo M, Restrepo B, Domínguez J. Estudio de tripanosomiasis americana en dos poblados indígenas de la Sierra Nevada de Santa Marta. CES Med. 2004;18:43-50.

36. Cantillo-Barraza O, Gómez-Palacio A, Salazar D, MejíaJaramillo AM, Calle J, Triana O. Distribución geográfica y ecoepidemiología de la fauna de triatominos (Reduviidae: Triatominae) en la Isla Margarita del departamento de Bolívar, Colombia. Biomédica. 2010;30:382-9. http://dx.doi. org/10.7705/biomedica.v30i3.272

37. Gutiérrez R, Angulo V, Aguilar F, Reyes A, Tarazona Z, Sandoval C. Aspectos ecológicos de la enfermedad de Chagas en la región nororiental de Colombia. En: Memorias del Curso taller internacional: Biología, epidemiología y control de tripanosomiasis americana y leishmaniosis. Ibagué: Universidad del Tolima; 2000.

38. Ramírez CJ, Jaramillo CA, del Pilar-Delgado M, Pinto NA, Aguilera G, Guhl F. Genetic structure of sylvatic, peridomestic and domestic populations of Triatoma dimidiata (Hemiptera: Reduviidae) from an endemic zone of Boyacá, Colombia. Acta Trop. 2005;93:23-9. http://dx.doi. org/10.1016/j.actatropica.2004.09.001

39. Turriago B. Evaluación de deltametrina como alternativa de control de Triatoma dimidiata y determinación de la prevalencia de Trypanosoma cruzi en perros en una región endémica para la enfermedad de Chagas en el departamento de Boyacá, Colombia (tesis). Bogotá: Universidad de los Andes; 2005.

40. Angulo V. Ensayo de estrategias de control y vigilancia de Triatoma dimidiata en Colombia. En: Memorias del Primer Taller Internacional sobre control de la enfermedad de Chagas, Bogotá: Universidad de los Andes; 2005. p. 91105.

41. Grisales N, Triana O, Angulo V, Jaramillo N, ParraHenao G, Panzera F, et al. Diferenciación genética de tres poblaciones colombianas de Triatoma dimidiata (Latreille, 1811) mediante análisis molecular del gen mitocondrial ND4. Biomédica. 2010;30:207-14. http://dx.doi.org/10.7705/ biomedica.v30i2.184

42. Gómez-Palacio A, Triana $\mathbf{O}$. Molecular evidence of demographic expansion of the Chagas disease vector Triatoma dimidiata (Hemiptera, Reduviidae, Triatominae) in Colombia. PLoS Negl Trop Dis. 2014;8:e2734. http://dx.doi. org/10.1371/journal.pntd.0002734

43. Lent $\mathbf{H}$, Wygodzinsky PW. Revision of the Triatominae (Hemiptera, Reduviidae), and their significance as vectors of Chagas' disease. Bull Am Mus Nat Hist. 1979;163:3. 
44. Dujardin J-P, Schofield J, Panzera F, Matías A, De La Riva J. Los vectores de la enfermedad de Chagas. Bruxelles: Académie Royale des Sciences d'Outre-Mer; 2002. Fecha de consulta: $1^{\circ}$ de marzo de 2015. Disponible en: http:// www.documentation.ird.fr/hor/fdi:010055328

45. Anderson RP, Gómez-Laverde M, Peterson AT. Geographical distributions of spiny pocket mice in South America: Insights from predictive models. Glob Ecol Biogeogr. 2002;11:131-41. http://dx.doi.org/10.1046/j.1466822X.2002.00275.x

46. Calderón Cl, Dorn PL, Melgar S, Chávez JJ, Rodas A, Rosales $\mathbf{R}$, et al. A preliminary assessment of genetic differentiation of Triatoma dimidiata (Hemiptera: Reduviidae) in Guatemala by random amplification of polymorphic DNApolymerase chain reaction. J Med Entomol. 2004;41:882-7. http://dx.doi.org/10.1603/0022-2585-41.5.882

47. Lyman DF, Monteiro FA, Escalante AA, Cordón-Rosales C, Wesson DM, Dujardin JP, et al. Mitochondrial DNA sequence variation among triatomine vectors of Chagas' disease. Am J Trop Med Hyg. 1999;60:377-86.

48. Bargues M, Guhl F, Mas-Coma S. Genetic characterization of domestic, peridomestic and sylvatic Triatoma dimidiata populations from Colombia by ribosomal DNA ITS-2 sequence analyses. Acta Trop. 2002;83:149.

49. Gómez-Palacio A. Diferenciación poblacional de Triatoma dimidiata capitata (Hemiptera: Reduvidae: Triatominae) de Colombia y su relación con la evolución molecular del complejo críptico dimidiata (tesis). Medellín: Universidad de Antioquia; 2012.

50. Jurberg J, Galvão C, Noireau F, Carcavallo RU, Rocha D da S, Lent H. Uma iconografia dos Triatomíneos (Hemiptera:Reduviidae). Rio de Janeiro: Editora Gama Filho; 2005.

51. Schofield CJ. Propuestas de estrategias para el control de Triatoma dimidiata en Colombia. En: Memorias del Primer Taller Internacional sobre control de la enfermedad de Chagas. Bogotá: Universidad de los Andes; 2005.

52. Barbosa SE, Dujardin JP, Soares RP, Pires HH, Margonari C, Romanha AJ, et al. Interpopulation variability among Panstrongylus megistus (Hemiptera: Reduviidae) from Brazil. J Med Entomol. 2003;40:411-20. http://dx.doi. org/10.1603/0022-2585-40.4.411

53. Esteban L. Variabilidad morfológica entre poblaciones de Triatoma dimidiata (Latreille 1811), procedentes de cuatro departamentos de Colombia (tesis). Medellín: Universidad Nacional de Colombia, sede Medellín; 2010.

54. Dujardin JP, Costa J, Bustamante D, Jaramillo N, Catalá S. Deciphering morphology in Triatominae: The evolutionary signals. Acta Trop. 2009;110: 101-11. http://dx. doi.org/10.1016/j.actatropica.2008.09.026

55. Bustamante DM, Monroy C, Menes M, Rodas A, SalazarSchettino PM, Rojas G, et al. Metric variation among geographic populations of the Chagas vector Triatoma dimidiata (Hemiptera: Reduviidae: Triatominae) and related species. J Med Entomol. 2004;41:296-301. http://dx.doi. org/10.1603/0022-2585-41.3.296

56. Catalá S, Sachetto C, Moreno M, Rosales R, SalazarSchetrino PM, Gorla D. Antennal phenotype of Triatoma dimidiata populations and its relationship with species of Phyllosoma and Protracta complexes. J Med Entomol. 2005;42:719-25. http://dx.doi.org/10.1093/jmedent/42.5.719
57. Arroyo CM, Esteban L, Catalá S, Angulo VM. Variación del fenotipo antenal de poblaciones del domicilio, peridomicilio y silvestres de Triatoma dimidiata (Hemiptera: Reduviidae) en Santander, Colombia. Biomédica. 2007;27:92-100. http:// dx.doi.org/10.7705/biomedica.v27i1.252

58. Pacheco-Tucuch FS, Ramírez-Sierra MJ, Gourbière S, Dumonteil E. Public street lights increase house infestation by the Chagas disease vector Triatoma dimidiata. PloS One. 2012;7:e36207. http://dx.doi.org/10.1371/journal.pone. 0036207

59. Zeledón R, Solano G, Zúñiga A, Swartzwelder JC. Biology and ethology of Triatoma dimidiata (Latreille, 1811). 3. Habitat and blood sources. J Med Entomol. 1973;10:36370. http://dx.doi.org/10.1093/jmedent/10.4.363

60. Zeledón R, Solano G, Burstin L, Swartzwelder JC. Epidemiological pattern of Chagas' disease in an endemic area of Costa Rica. Am J Trop Med Hyg. 1975;24:214-25.

61. Zeledón R, Montenegro VM, Zeledón O. Evidence of colonization of man-made ecotopes by Triatoma dimidiata (Latreille, 1811) in Costa Rica. Mem Inst Oswaldo Cruz. 2001;96:659-60. http://dx.doi.org/10.1590/S0074-02762001 000500012

62. Dumonteil E, Ruiz-Piña H, Rodríguez-Félix E, BarreraPérez M, Ramírez-Sierra MJ, Rabinovich JE, et al. Re-infestation of houses by Triatoma dimidiata after intradomicile insecticide application in the Yucatán peninsula, México. Mem Inst Oswaldo Cruz. 2004;99:253-6. http:// dx.doi.org//S0074-02762004000300002

63. Torres-Montero J, López-Monteon A, Dumonteil E, Ramos-Ligonio A. House infestation dynamics and feeding sources of Triatoma dimidiata in central Veracruz, México. Am J Trop Med Hyg. 2012;86:677-82. http://dx.doi. org/10.4269/ajtmh.2012.11-0746

64. Martínez-lbarra JA, Miguel-Álvarez A, ArredondoJiménez Jl, Rodríguez-López MH. Update on the biology of Triatoma dimidiata Latreille (Hemiptera: Reduviidae) under laboratory conditions. J Am Mosq Control Assoc. 2001;17:209-10.

65. Ventura-García L, Roura M, Pell C, Posada E, Gascón $\mathrm{J}$, Aldasoro E, et al. Socio-cultural aspects of Chagas disease: A systematic review of qualitative research. PLoS Negl Trop Dis. 2013;7:e2410. http://dx.doi.org/10.1371/ journal.pntd.0002410

66. Dumonteil E, Nouvellet P, Rosecrans K, Ramírez-Sierra MJ, Gamboa-León R, Cruz-Chan V, et al. Eco-bio-social determinants for house infestation by non-domiciliated Triatoma dimidiata in the Yucatán Peninsula, México. PLoS Negl Trop Dis. 2013;7:e2466. http://dx.doi.org/10.1371/ journal.pntd.0002466

67. Medina-Torres I, Vázquez-Chagoyán JC, RodríguezVivas RI, de Oca-Jiménez RM. Risk factors associated with triatomines and its infection with Trypanosoma cruzi in rural communities from the southern region of the State of México, México. Am J Trop Med Hyg. 2010;82:49-54. http:// dx.doi.org/10.4269/ajtmh.2010.08-0624

68. Hernández JL, Rebollar-Téllez EA, Infante F, Morón A, Castillo A. Indicators of infestation, colonization and infection of Triatoma dimidiata (Latreille) (Hemiptera: Reduviidae) in Campeche, México. Neotrop Entomol. 2010;39:1024-31. http://dx.doi.org/10.1590/S1519-566X2010000600027 
69. Polonio R, Ramírez-Sierra MJ, Dumonteil E. Dynamics and distribution of house infestation by Triatoma dimidiata in central and southern Belize. Vector Borne Zoonotic Dis. 2009;9:19-24. http://dx.doi.org/10.1089/vbz.2008.0002

70. Bustamante DM, Monroy MC, Rodas AG, Juárez JA, Malone JB. Environmental determinants of the distribution of Chagas disease vectors in south-eastern Guatemala. Geospat Health. 2007;1:199-211. http://dx.doi.org/10.4081/ gh.2007.268

71. Parra-Henao G, Quirós-Gómez O, Jaramillo-O N, Cardona ÁS. Environmental determinants of the distribution of Chagas disease vector Triatoma dimidiata in Colombia. Am J Trop Med Hyg. 2016;94:767-74. http://dx.doi.org/10.4269/ ajtmh.15-0197

72. Guzmán-Tapia Y, Ramírez-Sierra MJ, Escobedo-Ortegón J, Dumonteil E. Effect of Hurricane Isidore on Triatoma dimidiata distribution and Chagas disease transmission risk in the Yucatán Peninsula of México. Am J Trop Med Hyg. 2005;73:1019-25.

73. Ríos JF, Arboleda M, Montoya AN, Alarcón EP. Probable outbreak of oral transmission of Chagas disease in Turbo, Antioquia. Biomédica. 2011;31:185-95. http://dx.doi.org/10. 1590/S0120-41572011000200005

74. Ramírez JD, Montilla M, Cucunubá ZM, Flórez AC, Zambrano P, Guhl F. Molecular epidemiology of human oral Chagas disease outbreaks in Colombia. PLoS Negl Trop Dis. 2013;7:e2041. http://dx.doi.org/10.1371/journal. pntd.0002041

75. Soto H, Tibaduiza T, Montilla M, Triana O, Suárez DC, Torres MT, et al. Investigation of vectors and reservoirs in an acute Chagas outbreak due to possible oral transmission in Aguachica, Cesar, Colombia. Cad Saúde Pública. 2014;30:746-56. http://dx.doi.org/10.1590/0102$311 \times 00024013$

76. Nicholls RS. Informe de la consulta técnica en epidemiología, prevención y manejo de la transmisión de la enfermedad de Chagas como enfermedad transmitida por alimentos: la experiencia en Colombia. Rio de Janeiro; OPS; 2006.
77. Rojas de Arias A, Ferro EA, Ferreira ME, Simancas LC. Chagas disease vector control through different intervention modalities in endemic localities of Paraguay. Bull World Health Organ. 1999;77:331-9.

78. Gürtler RE, Yadón ZE. Eco-bio-social research on community-based approaches for Chagas disease vector control in Latin America. Trans R Soc Trop Med Hyg. 2015;109:91-8. http://dx.doi.org/10.1093/trstmh/tru203

79. Waleckx E, Cámara-Mejía J, Ramírez-Sierra MJ, CruzChan V, Rosado-Vallado M, Vázquez-Narváez S, et al. An innovative ecohealth intervention for Chagas disease vector control in Yucatán, México. Trans R Soc Trop Med Hyg. 2015;109:143-9. http://dx.doi.org/10.1093/trstmh/tru200

80. De Urioste-Stone SM, Pennington PM, Pellecer E, Aguilar TM, Samayoa G, Perdomo HD, et al. Development of a community-based intervention for the control of Chagas disease based on peridomestic animal management: An eco-bio-social perspective. Trans R Soc Trop Med Hyg. 2015;109:159-67. http://dx.doi.org/10.1093/trstmh/tru202

81. Organización Panamericana de la Salud. Grupo de trabajo OPS para consulta en planificación, operativa, estrategia y evaluación de etapas avanzadas del control antivectorial en enfermedad de Chagas. Montevideo: OPS; 2001.

82. Magnani C, Días JC, Gontijo ED. How health activities view man and how man rethinks them: An anthropological analysis of Chagas disease control. Cad Saúde Pública. 2009;25:1947-56. http://dx.doi.org/10.1590/S0102-311X20 09000900009

83. Red Chagas Colombia. Programa Nacional de Investigación para la Prevención, Control y Tratamiento Integral de la enfermedad de Chagas en Colombia 2012-2020. Fecha de consulta: 3 de diciembre de 2016. Disponible en: http://www. redchagascolombia.org/quienes-somos/presentacion. 